\title{
Neurogenin 1 Null Mutant Ears Develop Fewer, Morphologically Normal Hair Cells in Smaller Sensory Epithelia Devoid of Innervation
}

\author{
Qiufu MA, ${ }^{1}$ David J. Anderson, ${ }^{2}$ and Bernd FritzSCH ${ }^{3}$ \\ ${ }^{1}$ Dana Farber Cancer Institute, Boston, M A 02115 USA \\ ${ }^{2}$ California Institute of Technology, Pasadena, CA 91125 USA \\ ${ }^{3}$ Department of Biomedical Sciences, Creighton University, Omaha, NE 68178 USA
}

Received: 3 May 2000; Accepted: 11 May 2000; O nline publication: 15 August 2000

\begin{abstract}
The proneuronal gene neurogenin 1 (ngn 1 ) is essential for development of the inner-ear sensory neurons that are completely absent in ngn 1 null mutants. Neither afferent, efferent, nor autonomic nerve fibers were detected in the ears of ngn 1 null mutants. We suggest that efferent and autonomic fibers are lost secondarily to the absence of afferents. In this article we show that ngn 1 null mutants develop smaller sensory epithelia with morphologically normal hair cells. In particular, the saccule is reduced dramatically and forms only a small recess with few hair cellsalong a duct connecting the utricle with the cochlea. Hair cells of newborn ngn 1 null mutants show no structural abnormalities, suggesting that embryonic development of hair cells is independent of innervation. H owever, the less regular pattern of dispersal within sensory epithelia may be caused by some effects of afferents or to the stunted growth of the sensory epithelia. Tracing of facial and stato-acoustic nerves in control and ngn 1 null mutants showed that only the distal, epibranchial, placodederived sensory neurons of the geniculate ganglion exist in mutants. Tracing further showed that these geniculate ganglion neurons project exclusively to the solitary tract. In addition to the normal complement of facial branchial and visceral motoneurons, ngn 1 null mutants have some trigeminal motoneurons and contralateral inner-ear efferents projecting, at least temporarily, through the facial nerve. These data suggest
\end{abstract}

Correspondence to: Bernd Fritzsch, Ph.D. - Department of Biomedical Sciences. 2500 California Plaza. • O maha, NE 68178 USA. Telephone: (402) 280-2915; fax: (402) 280-5556; email: fritzsch@ creighton.edu that some neurons in the brainstem (e.g., inner-ear efferents, trigeminal motoneurons) require afferents to grow along and redirect to ectopic cranial nerve roots in the absence of their corresponding sensory roots.

Keywords: proneuronal genes, ear development, ganglion cell development, inner ear efferents, bH LH genes

\section{INTRO DUCTION}

Development of neuronsrequirescertain proneuronal basic helix-loop-helix ( $b H L H$ ) genes. These bHLH genes, first identified in fruit flies, have mammalian homologs such as M ash, M ath, N euroD, and the neurogenins (Lee 1997; Anderson 1999). The two neurogenins $n g n 1$ and $n g n 2$ are expressed in sensory neuron progenitors of the peripheral nervous system (PNS), upstream of N euroD and M ath3 ( Ma et al. 1997). Null mutations have shown that ngn 1 is essential for the formation of proximal, neural-crest-derived cranial sensory neurons( Ma et al. 1998). In contrast, ngn 2 null mutations initially lack distal, epibranchial placodederived cranial sensory neurons, though some seem to form later (Fode et al. 1998). In addition, data on ngn 1 null mutants suggest that inner ear sensory neurons are absent up to embryonic day 12 (E12; Ma et al. 1998). H owever, it is possible that some sensory neurons (Ruben 1967) may form later. It has been shown that this occurs in older ngn 2 null mutants with respect to geniculate ganglion neurons (Fode et al. 1998) and spinal ganglion neurons ( $M a$ et al. 1999b, b). In this study we show that no differentiated sensory 
neurons can be detected in E12, E14, E16, and P0 ngn 1 null mutant ears.

The likely absence of ganglion cells at any stage of ear development offers the opportunity to revisit the controversial issue of afferent influence on hair cell maturation. Numerous studies have highlighted the structural and functional independence of hair cell maturation in tissue culture of mice (van de Water 1983; Sobkowicz 1992; Rusch et al. 1998) and in chickens (Corwin and Cotanche 1989). Likewise, data in neurotrophin mutants with partial or complete absence of inner vation suggest that structural maturation of hair cells is independent of innervation (Fritzsch et al. 1997a; Silos-Santiago et al. 1997). H owever, none of these studies can rule out an inductive influence of sensory neurons on hair cell maturation before the former leave the otocyst wall (Carney and Silver 1983; Adam et al. 1998; Fritzsch et al. 1999). O ther studies have found incomplete physiologic maturation of hair cells in tissue culture (Sokolowski et al. 1993) and reduced size of sensory epithelia in neurotrophin receptor mutants (Schimmang et al. 1995). Absence of any sensory neuron formation could thus settle this long-standing discussion (Fritzsch et al. 1998b), at least for the hair cells of the mammalian inner ear. Our data show normal development of hair cells, but not of sen soryepithelia, in ngn 1 null mutants, thus supporting the hypothesis of the autonomy of hair cell differentiation in rodents until birth.

In addition to the afferent innervation by inner ear sensory neurons, the ear also receives an efferent innervation from cells in the hindbrain (Fritzsch 1999) and an autonomic inner vation from the superior cer vical ganglion (Shibamori et al. 1994). H ow these fibers reach the ear during development is unclear. Based on a correlative loss of efferent and afferent fibers to sensory epithelia in various neurotrophin mutants, it is possible that efferent fibers navigate within the ear along afferent fibers to reach the various sensory epithelia (Fritzsch et al. 1998a). H owever, this correlative loss is true only for efferent fibers going to the sensory epithelia. Within sensory epithelia, efferent fibers seem to have some capacity to reach targets without afferents. For example, efferent fibers can reach the outer hair cells in the apex of the cochlea in BDNF and trkB null mutants (Fritzsch et al. 1997a). H owever, efferents do not reach the outer hair cells in the basal turn of NT-3 null mutants (Fritzsch et al. 1997b). It has been suggested that efferent fibers may be guided to outer hair cells at least in part by differential expression of neurotrophins (Wiechers et al. 1999). In addition, the absence of efferents appears to dramatically affect the maturation of the cochlea, causing permanent threshold elevations (Walsh et al. 1998) that are not mediated through the nicotinic $\alpha 9$ receptor (Vetter et al. 1999; Zuo et al. 1999).
The absence of afferents and their processesin ngn 1 null mutants allows us to address the question of whether autonomic and efferent fibers are able to reach the ear and the various sensory epithelia without the aid of afferents. Our data suggest that many inner ear efferents form normally in ngn 1 null mutant mice, probably because of functional redundancy of ngn 1 and $n g n 2$ in the basal plate ( $M a$ et al. 1997). H owever, some inner ear efferent neurons redirect their axons and project temporarily through the facial nerve in the absence of their corresponding sensory neurons. A preliminary account of these data has been published as an abstract ( Ma et al. 1999a).

\section{MATERIAL AND METHODS}

All handling of animals was approved by the Dana Farber Cancer Institute's animal care committee in agreement with $\mathrm{NIH}$ guidelines. ngn 1 null mutant mice were obtained from breeding of heterozygotic ngn 1+ / - mice as previously described ( Ma et al. 1998, 1999b). Litters were collected from timed pregnant mice at embryonic day 12 (E12) , E14, E16, and at birth (E19/ P0) . Femaleswere killed byvertebral dislocation. The embryos and newborns were anesthetized by hypothermia until immobile and perfusion-fixed in $4 \%$ paraformaldehyde.

Genotyping was performed by polymerase chain reaction (PCR) as described in Ma et al. (1998) using the following pair of primers: 5'TGGTGTCGTCGGGGAACGA3' and 5'AAGGCCGACCTCCAAACCTC3' for wildtype allele ( $0.4 \mathrm{~kb})$; 5'GATCG GCCATT GAACAAGATGGATTGCA3' and 5'AGCTCTTCAGCAATATCACGGGTAGCCA3' for mutant allele. N gn 1-/ - animals will be referred to as null mutants. Wildtype and ngn $1+/$ - littermates will be referred to as control animals.

\section{TEM and SEM of ears}

Ears of each five ngn 1 null mutants and five control littermates (P0) were dissected, viewed, and photographed as whole mounts. The length of each of five flat-mounted cochleae was measured using I magePro (Media Cybernetics, Silver Springs, MD) software (Table 1). In addition, drawings of these ears were made with a camera lucida (Fig. 1). Subsequently, ears were osmicated, dehydrated, and embedded in epoxy resin for transmission electron microscopy (TEM). Light microscopic analysis was performed on semithick sections and ultrathin sections were examined using a Phillips TEM. The remaining five ears were critical point dried, mounted, sputter coated, and viewed in a Jeol scanning electron microscope (SEM).

$\mathrm{H}$ air cell were counted on $100-\mu \mathrm{m}$-long stretches 


\begin{tabular}{|c|c|c|c|c|c|}
\hline \multicolumn{6}{|c|}{ Reduction of length or width of sensory epithelia in P0 ngn1 null mutants } \\
\hline U tricle & AVC & PVC & $\mathrm{HC}$ & Saccule & Cochlea \\
\hline $\begin{array}{l}\text { ngn1 null mutant } \\
* 554 \pm 35 \mu \mathrm{m} \\
* * 286 \pm 28 \mu \mathrm{m}\end{array}$ & $* 290 \pm 62 \mu \mathrm{m}$ & $* 343 \pm 58 \mu \mathrm{m}$ & $* 229 \pm 53 \mu \mathrm{m}$ & $\begin{array}{r}* 158 \pm 89 \mu \mathrm{m} \\
* * 115 \pm 74 \mu \mathrm{m}\end{array}$ & $* 2597 \pm 208 \mu \mathrm{m}$ \\
\hline $\begin{array}{l}\% \text { Reduction } \\
* 11-* * 33 \%\end{array}$ & $36 \%$ & $30 \%$ & $43 \%$ & $* 76-* * 80 \%$ & $49 \%$ \\
\hline $\begin{array}{l}\text { ngn1 wildtype } \\
* 621 \pm 41 \mu \mathrm{m} \\
* * 424 \pm 32 \mu \mathrm{m}\end{array}$ & $* 455 \pm 68 \mu \mathrm{m}$ & $* 484 \pm 75 \mu \mathrm{m}$ & $* 401 \pm 67 \mu \mathrm{m}$ & $\begin{array}{r}* 609 \pm 59 \mu \mathrm{m} \\
* * 564 \pm 46 \mu \mathrm{m}\end{array}$ & $5114 \pm 368 \mu \mathrm{m}$ \\
\hline
\end{tabular}

Mean and standard deviation of 5 ears: ${ }^{*}$ and $* *$ indicated longest and shortest axes, respectively. AVC = anterior vertical canal; PVC $=$ posterior vertical canal; $\mathrm{HC}=$ horizontal canal

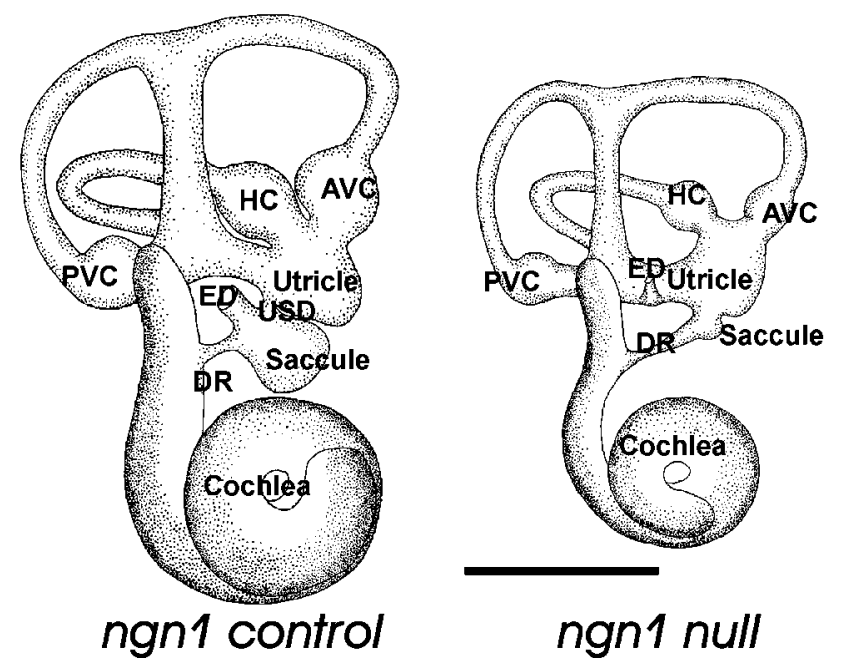

FIG . 1. Dissected ears of PO ngn 1 null mutant and littermate control mice showing the overall reduction in size of the mutant ear. In addition, the cochlea shows only $1 \frac{1}{4}$ turn in the mutant but $1 \frac{1}{2}$ turn in the control littermate. In the control mutant, the large saccular recess is separated from the utricle by the utriculo-saccular duct (USD) and from the cochlea by the ductus reuniens (DR). In contrast, in ngn1 null mutants, the very small saccular recess is at the ductus reuniens (DR) and no distinct utriculosaccular duct exists. O ther sensory epithelia are also smaller, but not as much as the saccule. $\mathrm{ED}=$ endolymphatic duct, $\mathrm{AVC}=$ anterior vertical canal, $\mathrm{HC}=$ horizontal canal, PVC $=$ posterior vertical canal, scale bar equals $1 \mathrm{~mm}$.

of all five cochleae prepared for SEM. SEM photographs at $1000 \times$ were taken of the basal, middle, and apical areas of each of the five cochleae (Fig. 3). I mages were chosen from the middle part of each area, except for the apex where lack of differentiation forced us to choose a more basal stretch of hair cells. In cases were debris precluded analysis (three out of 15 samples), we examined somewhat more basal or apical stretches. Hair cells were counted directly on the photographs and their mean and standard deviation are given in Table 2. Total mean was calculated from the basal, middle, and apical turn and multiplied by the total

\begin{tabular}{lccccc}
\hline \multicolumn{5}{c}{ TABLE 2 } \\
\hline \multicolumn{3}{c}{ Numbers of hair cells per 100} & $\mu \mathrm{m}$ of cochlea \\
\cline { 2 - 3 } \cline { 5 - 6 } & \multicolumn{2}{c}{ Wildtype } & & \multicolumn{2}{c}{ Mutant } \\
\cline { 2 - 3 } \cline { 5 - 6 } & OHC & IHC & & OHC & IHC \\
Base & $72 \pm 8$ & $23 \pm 3$ & & $74 \pm 9$ & $23 \pm 3$ \\
Middle & $78 \pm 9$ & $23 \pm 4$ & & $80 \pm 12$ & $36 \pm 7$ \\
Apex & $82 \pm 9$ & $23 \pm 5$ & & $101 \pm 15$ & $46 \pm 9$ \\
Mean & 77 & 23 & & 85 & 35 \\
Total & 3930 & 1170 & & 2210 & 910 \\
\hline
\end{tabular}

Mean and standard deviation of 5 wildtype control and 5 ngn 1 null mutants. Total hair cells were calculated by multiplying the mean of hair cells with the length of the cochlea.

length of the ngn 1 null and wildtype control mice cochleae (Table 1) to obtain an estimate of total hair cells per cochlea (Table 2 ).

\section{Tract tracing}

Four E12, two E14 and E16, and six P0 ngn 1 null mutants and comparable numbers of control littermates received Dil injections into either the facial nerve, the inner ear, or the inner ear and facial nerve combined. Dil was applied via soaked filter strips (Fritzsch and Nichols 1993) and diffused for three (E12 embryos), four (E14, E16), or five days (P0) at $37^{\circ} \mathrm{C}$. Brains of E12 mice were dissected, mounted flat, and viewed with an epifluorescence microscope. Subsequently, these brains as well as those of the E14, E16, and newborn mice were embedded in $12 \%$ gelatin and cut to $80-\mu \mathrm{m}$ thickness with a vibratome. Sections were mounted and viewed with an epifluorescence microscope.

O ne E12 and three P0 ngn 1 null mutants, plus one control littermate of E12 and P0, were injected with Dil closeto the left rhombomere 4 and 5 of the brainstem (E12) and near the pontine flexure (P0) in order to label all afferent and efferent fibers (Fritzsch and 
Nichols 1993). The ipsilateral and contralateral ears, facial nerve, and vestibular ganglia were dissected and viewed with an epifluorescence microscope. The brainstem was embedded in gelatin and sectioned as described above.

Immunocytochemistry for nerve fibers using acetylated tubulin

Three P0 ngn 1 null mutant ears and three control ears were dissected and underwent immunocytochemical detection of acetylated tubulin (Fritzsch et al. 1997b). Briefly, ears were defatted and incubated for four days with a primary anti-acetylated tubulin antibody (Sigma), followed by a secondary antibody conjugated to horseradish peroxidase (HRP). Diaminobenzidine was used as a substrate. Whole mounts of the entire ear or specific sensory epithelia were examined using bright field and differential interference contrast (DIC) light microscopy.

\section{RESU LTS}

\section{O rganization of the ear in ngn1 null mutants}

Comparison of the inner ears of PO ngn 1 mutant mice with those of control littermates revealed smaller inner ears but other wise showed little deviation from normal development (Fig. 1). Sensory epithelia were reduced in length or width (Fig. 1, Table 1). For example, the cochlea in the ngn 1 null mutant was only about half as long and the saccule formed only a ver y small extension on the utricle leading to the ductus reuniens connecting to the cochlea (Fig. 1). No utriculosaccular duct was found in ngn 1 null mutants, and the sensory epithelium of the utricle and saccule were almost continuous (Fig. 1). In addition, the cochlea showed only $1 \frac{1}{4}$ turn in ngn 1 null mutants but $1 \frac{3}{4}$ turn in control littermates (Fig. 1). ngn 1 null mutants had no modiolus in the center of the cochlea; instead there was a large empty space with a more narrow cochlear turn (Figs. 1 and 2). Other sensory epithelia were reduced in length and/or width by $10-40 \%$ (Table 1) but appeared otherwise normal (Figs. 1 and 2).

Examination of the whole-mounted ears of the $n g n 1$ null mutant reacted for acetylated tubulin revealed no nerve fibers (Fig. 2). Likewise, no afferent or efferent fibers to the ear could be labeled with Dil applied to the brainstem in E12, E14, E16, and P0 ngn 1 null mutants (data not shown). In contrast, we could trace afferents from their respective ganglia and efferents in both the intraganglionic bundles of the cochlea and the vestibular sensory epithelia of control littermates using acteylated tubulin immunocytochemistry (Fig. 2) and Dil. Control PO littermates had thin fibers around capillaries inside as well as outside the sensory epithelia. These fibers were visible only with antitubulin immunocytochemistry which suggests that they are not derived from the brainstem and likely represent autonomic fibers (data not shown) from the superior cervical ganglion (Shibamori et al. 1994). Acetylated tubulin immunocytochemistry labeled kinocilia of hair cells equally in both $n g n 1$ null mutants and control mice (Fig. 2). This suggests that the absence of nerve fiber labeling cannot be attributed to incomplete immunocytochemical reaction for acetylated tubulin. The labeling of the kinocilia in conjunction with differential interference contrast microscopy allowed us to define the boundaries of sensory epithelia and to measure the length or width of the various sensor y epithelia (Table 1, Fig. 2). These data suggest that hair cells are present throughout the sensory epithelia.

Scanning electron micrographs (SEM) confirmed this impression. We found that hair cells in the cochlea showed a less regular alignment within more rows in the middle and apical turn of ngn 1 null mutants (Fig. 3). Interestingly, hair cells were organized into the typical three rows of outer hair cells and the one row of inner hair cells only in the basal third of the cochlea in ngn 1 null mutants (Fig. 3). H owever, the apical part of the middle turn and the apical turn showed up to five rows of outer hair cells (OHCs) and two rows of inner hair cells (IHCs; Fig. 3d,f,h). H air cell counting in SEM micrographs of selected areas of ngn 1 null mutants showed comparable numbersin the basal turn (Table 2). The middle turn showed an increase of approximately $3 \%$ of OHCs and $57 \%$ of IHCs over control mice. Only the basal part of the apex could be counted this way (Fig. $3 g$ ) and showed a $23 \% \mathrm{OHC}$ and $100 \% \mathrm{IHC}$ increase. Even this increased number of hair cells in the apical half of the cochlea did not fully compensate for the $50 \%$ overall reduction in length of the entire cochlea of ngn 1 null mutants. Multiplying the mean of hair cells per cochlea with the length of the cochlea (Table 2) gives an overall reduction of approximately $22 \% \mathrm{IHCs}$ and $44 \% \mathrm{OHCs}$ in ngn 1 null mutants. This suggests that ngn 1 null mutants reduce the longitudinal growth of the middle and apical turn and regroup the fewer remaining inner and outer hair cells into more rows on a shortened and more steeply curved cochlea (Figs. 1 and 3).

We found similar packing density of hair cells in the vestibular sensory epithelia of ngn 1 null mutants and wildtype control littermates (Fig. 4a,b). Together with the reduced size of sensory epithelia (Table 1), this suggests a reduction of $20 \%$ (utricle) to $80 \%$ ( saccule) of hair cells (Fig. 4a,b inset).

Detailed light and transmission electron microscopy showed that hair cells and supporting cells differentiated normally despite the complete absence of any 

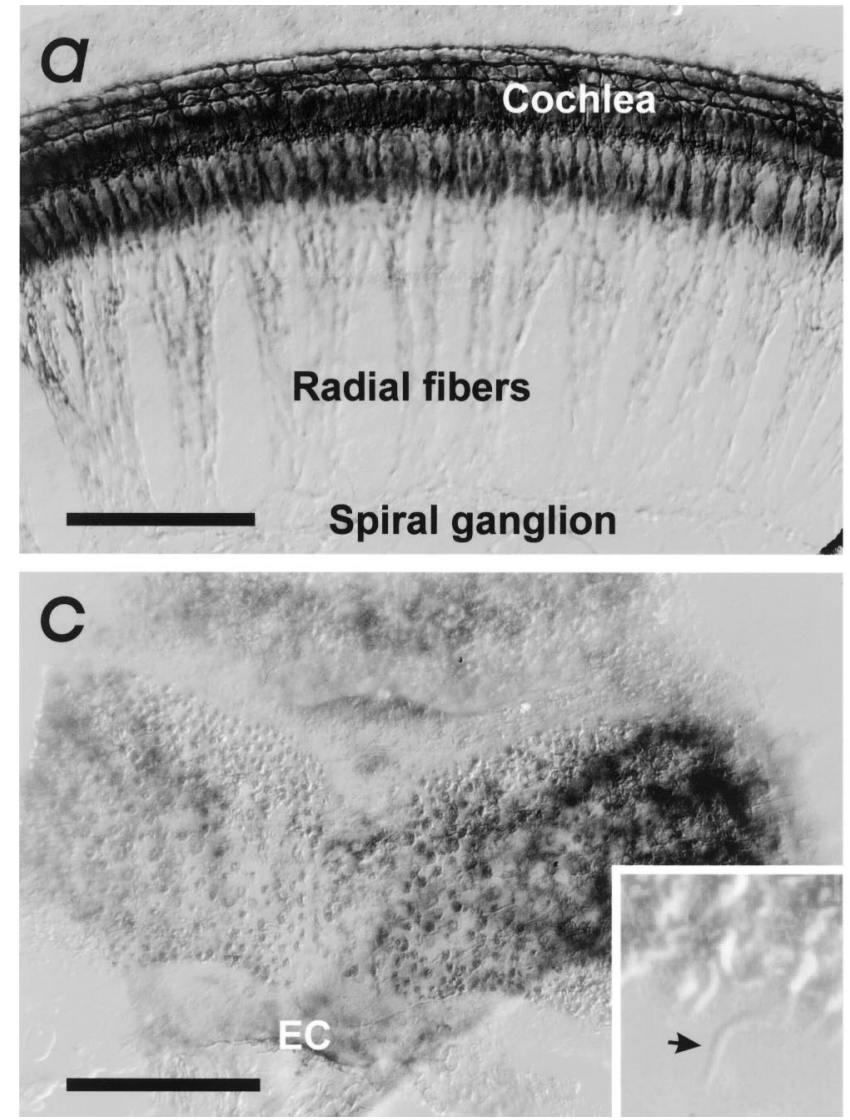

FIG . 2. Whole mounts showing nerve fibers and sensory epithelia of P0 ngn 1 null mutant $(\mathbf{b}, \mathbf{d})$ and control littermate $(\mathbf{a}, \mathbf{c})$. In the cochlea, spiral sensory afferents and efferent fibers are visible as radial fibers and spiraling fibers in the modiolus (a), but no fibers are labeled in the cochlea of ngn 1 null mutants and the modiolus is a central hole (b). Likewise, no fibers are visible to the posterior vertical canal sensory epithelium in ngn 1 null mutants (d), but they are visible

nerve fibers (Fig. 5). For example, in the cochlea we could distinguish inner and outer hair cells, Deiter's cells, and Pillar cells (Fig. 5). In the vestibular sensory epithelia we tentatively identified two types of hair cells based on hair cell intrinsic criteria. In the past, type I and type II vestibular hair cells were differentiated based on the presence or absence of a calyx formed by afferent fibers. However, a recent study identified distinguishing features intrinsic to hair cells ( Rusch et al. 1998). In agreement with these criteria, the nuclei of the two vestibular hair cell types showed differences in heterochromatin organization and in the ratio of the diameter of stereocilium to that of kinocilium in various planes of sections in adjacent hair cells (Fig. 5). Despite the absence of any fiber contact at hair cells, synaptic vesicles surrounding synaptic ribbons floating inside the cytoplasm and occasionally near the plasma membrane were found in hair cells (Fig. 5a).

In summar $y$, data on the ngn 1 null mutant ears show they are never innervated. Hair cells and supporting cells that develop normally in sensory epithelia are
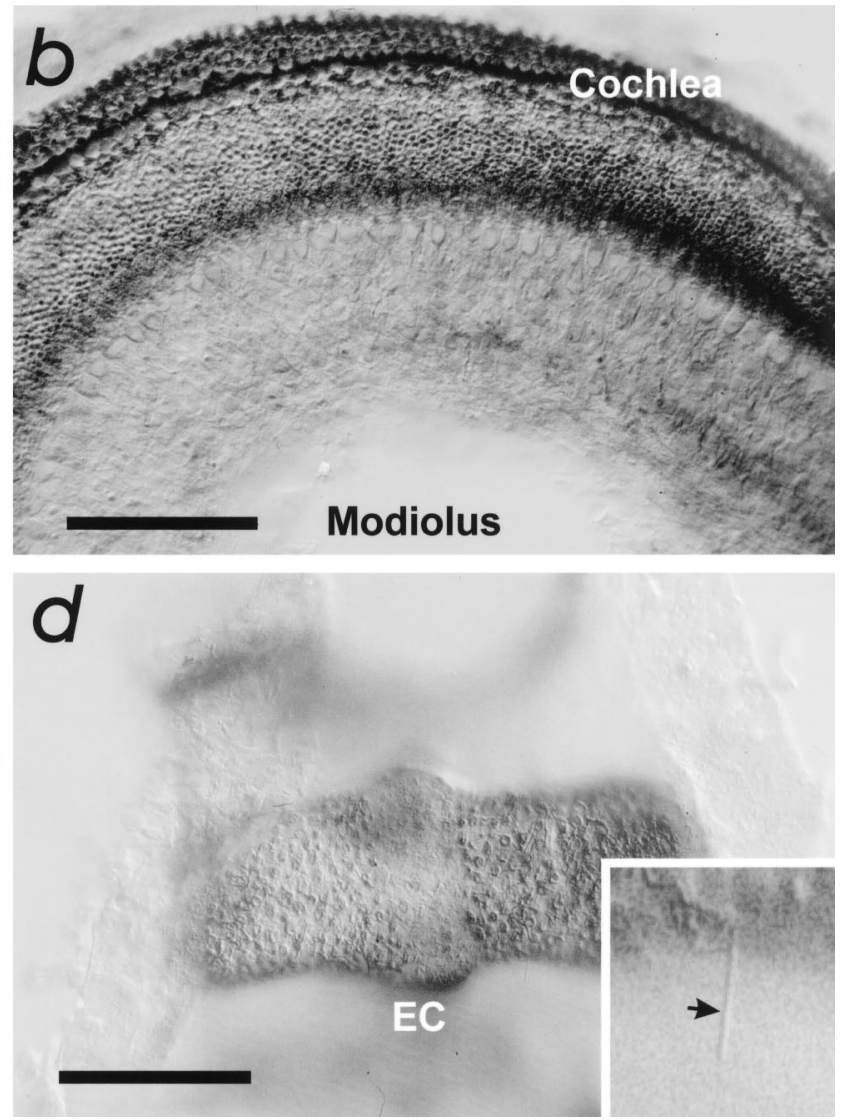

out of focus in the control littermate (c). Kinocilia of the hair cells are immunopositive for acetylated tubulin (insert, arrow) showing that the differences in nerve fiber immunocytochemistry are related to absence of all nerve fibers in the ngn 1 null mutant ear. N otice the reduced length in the canal sensory epithelia $(\mathbf{c}, \mathbf{d})$. EC = eminentia cruciatum, scale bar equals $100 \mu \mathrm{m}$.

variably reduced in length or width. The total number of hair cells in the cochlea appears to be reduced to $61 \%$ of that of control animals (Table 1). These data suggest that embryonic morphological differentiation of vestibular and cochlear hair cells occurs in the complete absence of innervation in vivo.

\section{Afferents and efferents to the inner ear and the facial nerve}

Large injections of Dil into the brainstem filled all afferents and efferents emanating from rhombomere 4. In wildtype and heterozygotic control littermates, these injections filled the facial branchial motoneuron axons running in the facial nerve (Fig. 6), the facial visceral motoneuron axons plus visceral and somatic afferents of the geniculate ganglion running in the intermediate nerve, and inner ear afferents and efferents. In contrast, comparable injections in littermate ngn 1 null mutants labeled only facial branchial motoneuron axons, facial visceral motoneuron axons, and 

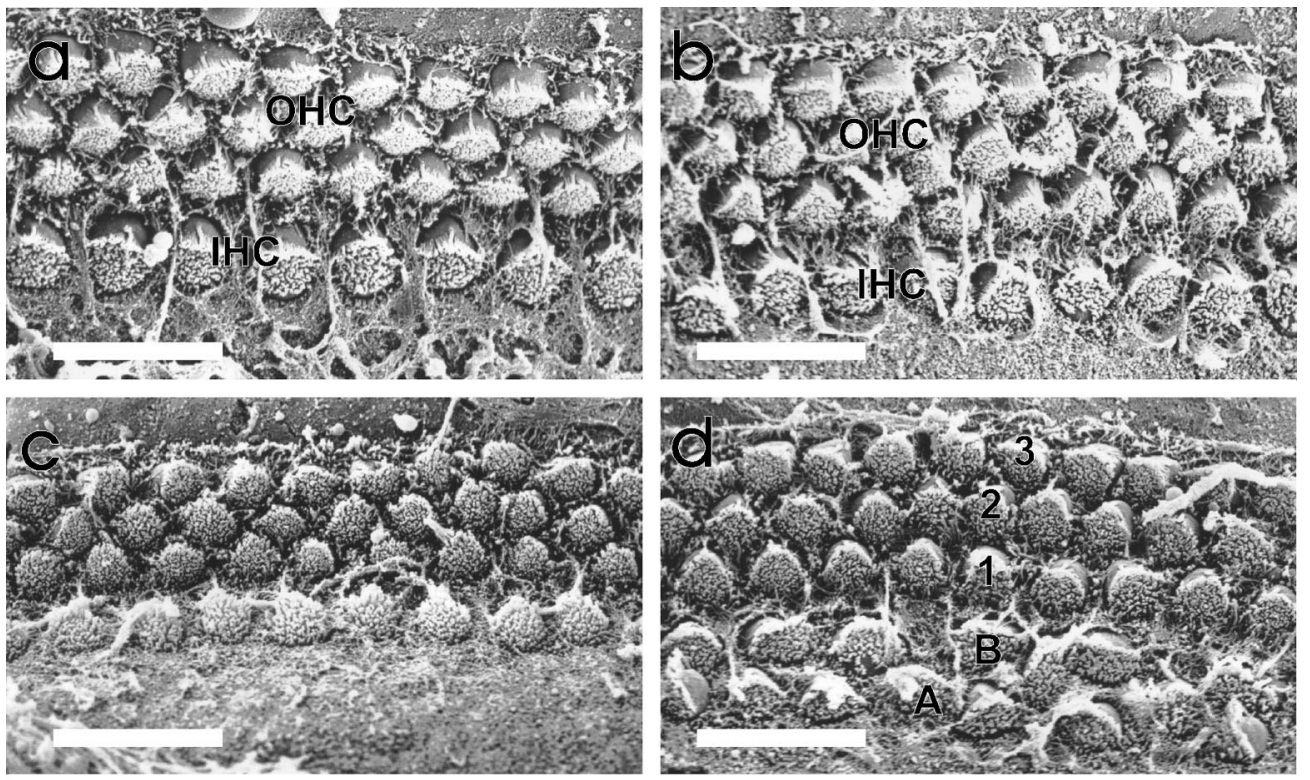

FIG. 3. Scanning electron micrographs compare the hair cell organization in the cochlea of newborn wildtype control littermate $(\mathbf{a}, \mathbf{c}, \mathbf{e})$ and ngn 1 null mutants (b, d, f, g, h). Images were taken from the middle part of the basal third, the middle part of the middle third, and the
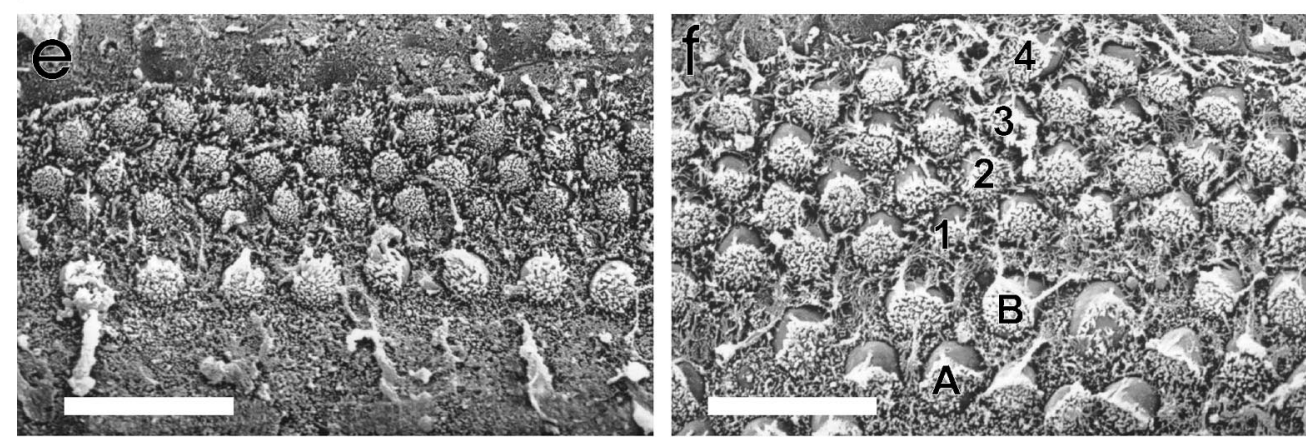
basal part of the apical third of the cochleae. O uter $(\mathrm{OHC})$ and inner hair cells $(\mathrm{IHC})$ of the cochlea show a largely comparable packing density in the basal turn of ngn 1 null mutant (a) and control animals (b). In the middle turn, up to two irregular rows of $\operatorname{IHCS}(A, B)$ are found in the mutant (d) but not in the wildtype littermate (c). In the apical turn, the ngn1 null mutant cochlea shows two
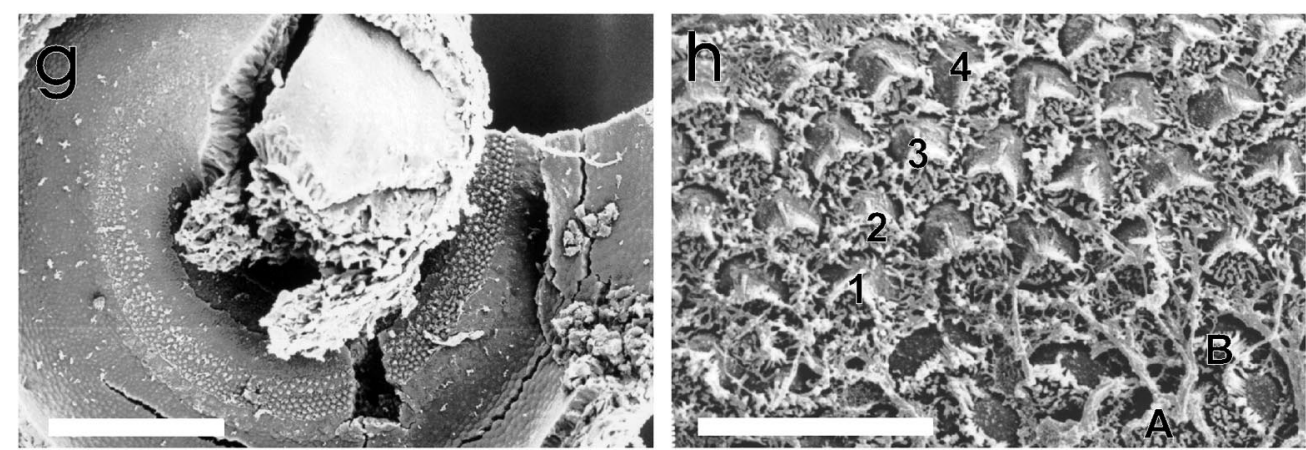
irregular rows of IHCS $(A, B)$ and four to five rows of $\mathrm{OHCs}(\mathbf{f}, \mathbf{h}$; taken from different ngn 1 null mutants), whereas the control littermate shows one row of IHC and three rows of $\mathrm{OHCs}(\mathbf{e})$. The overview of the apex of a ngn1 null mutant (g) shows the disorganization of the hair cells and the absence of hair cell apical specialization in the apical tip. (a-f, h) scale bar equals $10 \mu \mathrm{m}$; (g) scale bar equals $100 \mu \mathrm{m}$.

facial visceral afferent fibers (Fig. 6). Thus, no sensory neurons could be labeled in either the vestibular or the cochlear ganglion, and no efferent fibers reached the ear in ngn 1 null mutants at E12, E16, and P0 (Figs. 6 and 7). The complete absence of vestibular and proximal, neural-crest-derived, geniculate sensory neurons selectively revealed the trajectory of the intermediate nerve from a smaller geniculate ganglion toward the brain (Fig. 6). The large vestibular ganglion in control littermates (Fig. 6) camouflages these fibers of the intermediate nerve.

Selective application of Dil to the facial nerve in E12 control littermates showed facial branchial moto- neurons in rhombomeres 4-6, apparently migrating along the floor plate as previously described (Altman and Bayer 1981; Fritzsch and Nichols 1993; Studer et al. 1996). In addition, facial visceral motoneuronswere labeled in rhombomere 5 (Fritzsch and Nichols 1993; Bruce et al. 1997) and visceral afferents could be traced into the solitary tract (Figs. 6 and 7). A few somatic afferents that ran in the descending trigeminal tract were also seen. Application of Dil to the inner ear labeled inner ear efferent neurons bilaterally in rhombomere 4, with numerous fibers crossing the floor plate of this rhombomere (Fig. 7; insert). In addition, inner ear afferent fibers were labeled run- 

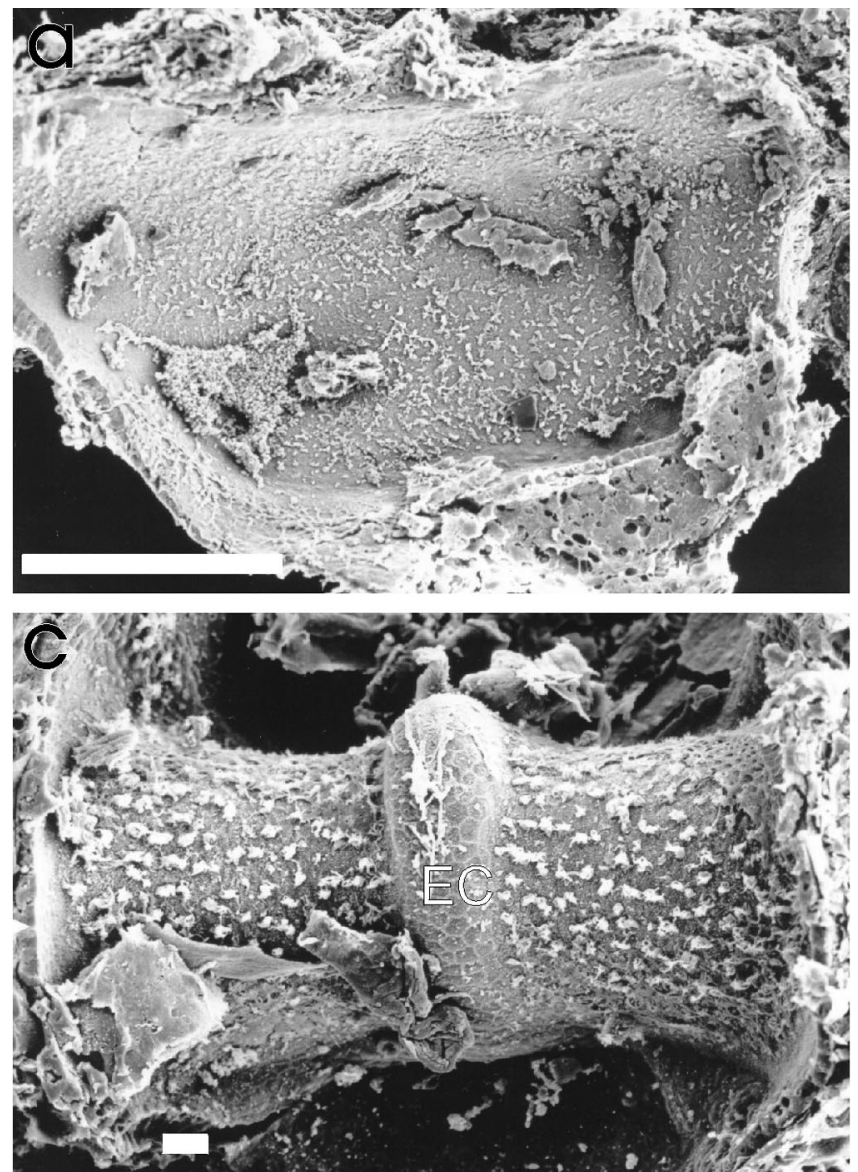

FIG . 4. Scanning electron micrographs compare the hair cell organization in the saccule $(\mathbf{a}, \mathbf{b})$ and anterior vertical canal $(\mathbf{c}, \mathbf{d})$ of control $(\mathbf{a}, \mathbf{c})$ and ngn 1 null mutant littermates $(\mathbf{b}, \mathbf{d})$. Compared with the control littermate, the saccule of ngn 1 null mutants is much reduced in length and width (compare $\mathbf{a}$ and $\mathbf{b}$ inset, both taken at

ning along the vestibular and cochlear nuclei toward the cerebellum and the obex region (Figs. 6 and 7). Dil application to both the inner ear and the facial nerve labeled both facial and inner ear afferents and efferents combined (Figs. 6 and 7).

In contrast to control littermates, after Dil application to the combined facial nerve and inner ear, we could not label inner-ear afferents in E12 ngn 1 null mutants, and no somatic afferents could be traced to the descending trigeminal tract (Figs. 6 and 7). In fact, the only afferents labeled after any application to the facial nerve with or without involving the inner ear were afferents entering through the intermediate nerve and running in the solitary tract (Figs. 6 and 7). In contrast, facial motoneurons were labeled in ngn 1 null mutants with intensity and distribution similar to those in control littermates (Fig. 7). Dil applications to the inner ear alone did not label any efferent neurons in ngn 1 null mutants. However, applications to the facial nerve labeled not only ipsilateral facial motoneurons but also several motoneurons on the
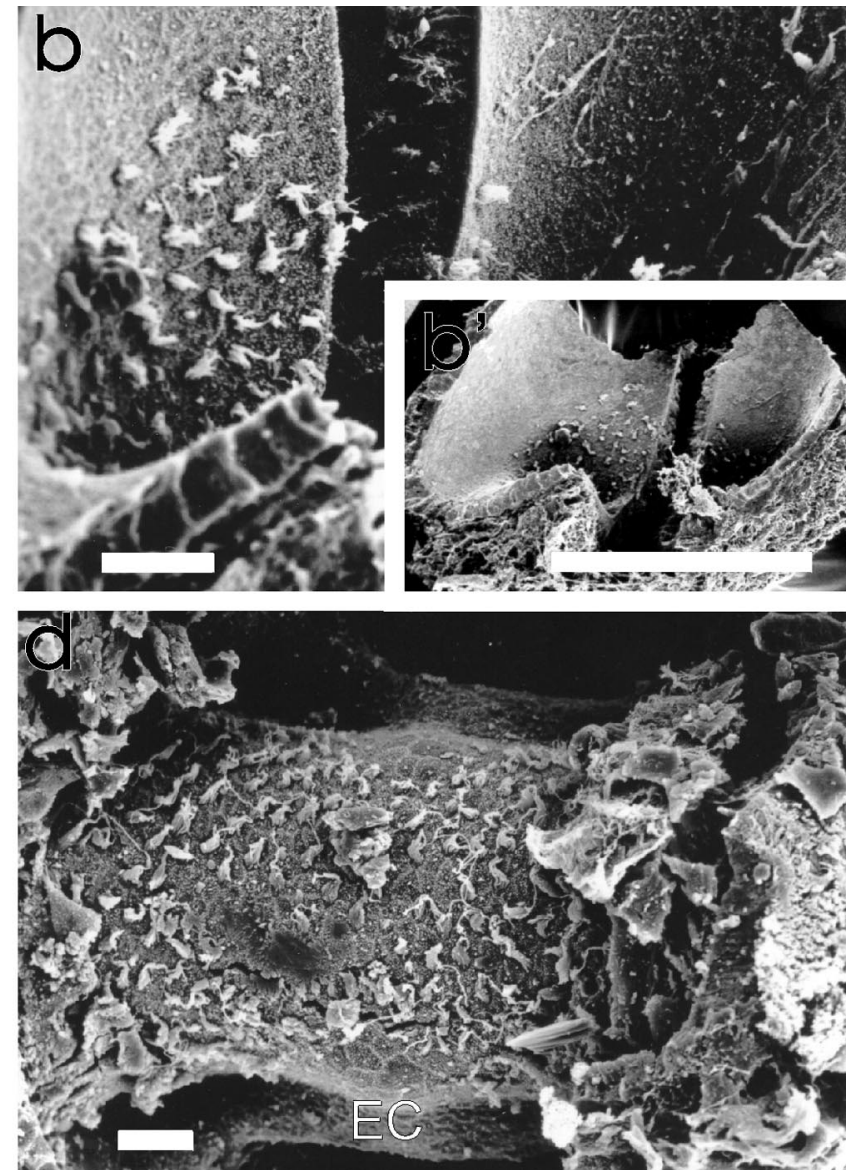

the same magnification) and shows fewer hair cells in a very small recess (b). The semicircular canal epithelium is shorter (compare magnification in $\mathbf{c}, \mathbf{d}$ ) and shows a less distinct eminentia cruciatum $(E C)$ in ngn1 null mutants than in control littermates. (b, c, d) Scale bar equals $10 \mu \mathrm{m}$; (a, b inset) scale bar equals $100 \mu \mathrm{m}$.

contralateral side of rhombomere 4 (Figs. 7 and 8). Such labeling was only rarely obser ved in ngn 1 control littermates. However, many more contralateral cells in a comparable distribution were labeled in rhombomere 4 of control littermates after Dil application to the inner ear alone or to the inner ear and facial nerve (Figs. 7 and 8). Nevertheless, the few contralateral cells labeled in ngn 1 null mutants after facial nerve applicationsclosely resembled the numerousinner ear efferents of control littermates in both their distribution pattern and morphology (Figs. 7 and 8). As with inner ear efferents of control littermates, these cells had fibers directed toward the contralateral intermediate nerve, and cells were found migrating toward the inferior olive (Fig. 8). In E16 ngn 1 null animals we could trace these contralateral fibers to cells positioned in the area of the vestibular efferents and a few cells in the superior olivary complex (Fig. 8). Fibers of these cells could be traced bilaterally to the inferior vestibular nucleus where they seemed to terminate. Several fibers could al so be traced toward the contralat- 

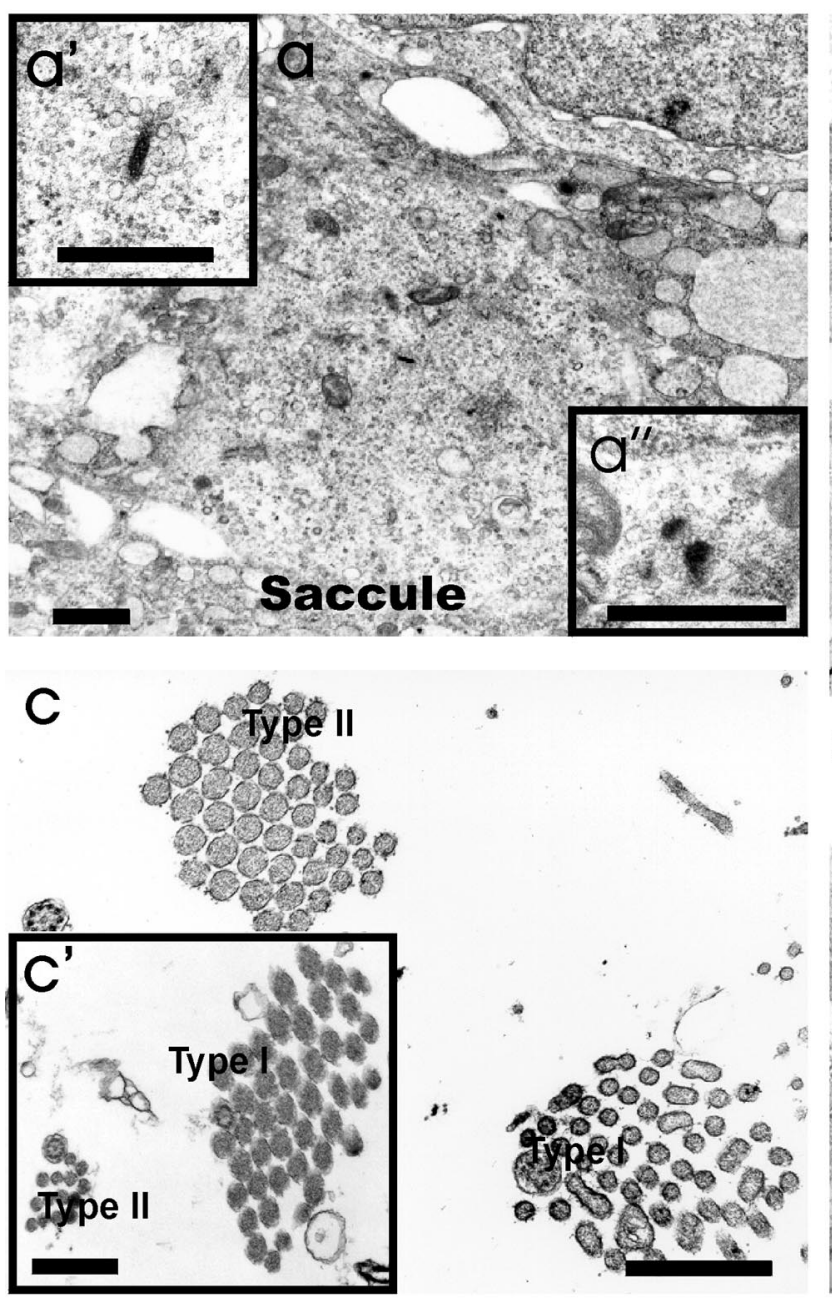

FIG. 5. Transmission electron micrographs show the organization of hair cells in the saccule (a), utricle (b, c), and cochlea (d) of P0 ngn1 null mice. N ote that the overall organization of sensory epithelia is rather normal and hair cells (HC), including inner (IHCs) and outer hair cells $(\mathrm{OHCs})$, can be distinguished from supporting cells such as pillar cells. Higher magnification shows the presence of presynaptic

eral facial nerve root. Only an occasional fiber was found to cross in the floor plate of rhombomere 4 of P0 null mutants and none were found after facial nerve applications in control littermates.

Application of Dil to the facial nerve of E12 ngn 1 null mutantslabeled not only facial motoneurons ( Fig. 7) but also labeled contralateral, inner ear efferentlike neurons in rhombomeres 4-6 (Figs. 7 and 8). We also labeled many trigeminal motoneurons in rhombomeres 2 and 3 after facial nerve application in E12 ngn 1 null mutants (Figs. 6 and 7). The dense clustering of these labeled motoneurons precluded proper counting. Only a very few motoneurons (less than 10) in the caudal part of rhombomere 3 were labeled in E12 control littermates, and no trigeminal motoneurons were labeled in E14, E16, and P0 ngn 1 null
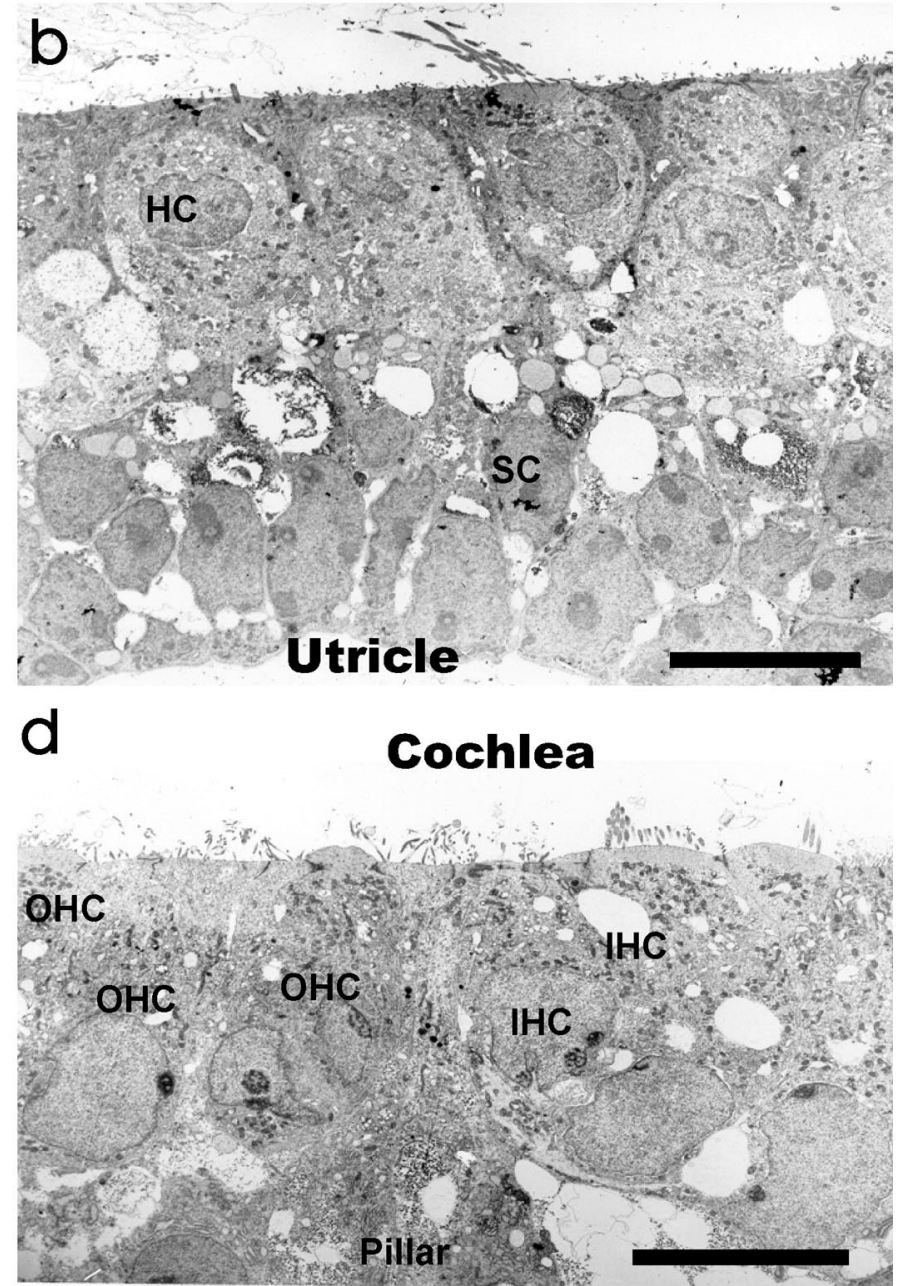

bars surrounded by synaptic vesicles (a', $\left.\mathbf{a}^{\prime \prime}\right)$ inside hair cells. Closer examination of vestibular hair cells shows that neighboring hair cells have different-sized stereocilia (c, $\left.\mathbf{c}^{\prime}\right)$. They are consistently either as large as the kinocilium (Type I) or much thinner than the kinocilium (Type II). (a, b, d) Scale bar equals $10 \mu \mathrm{m} ;\left(\mathbf{a}^{\prime}, \mathbf{a}^{\prime \prime}, \mathbf{c}, \mathbf{c}^{\prime}\right)$ scale bar equals $1 \mu \mathrm{m}$.

mutants after facial nerve applications. Trigeminal motoneurons apparently projected across one or two rhombomere boundaries to exit the brainstem through the facial nerve (Fig. 7).

In summary, ngn 1 null mutants retain only the distal, epibranchial, placode-derived visceral afferents in the facial and stato-acoustic nerve that project to the solitary tract (Fig. 6). In contrast, many more motoneuron populations project, at least temporarily, through the facial nerve in the absence of sensory neuron formation in proximal cranial ganglia of $n g n 1$ null mutants. Thus, we find contralateral cells in rhombomere 4 that resemble inner ear efferents and trigeminal motoneurons in rhombomeres 2 and 3 are labeled from the facial nerve in E12 ngn 1 null mutants. 

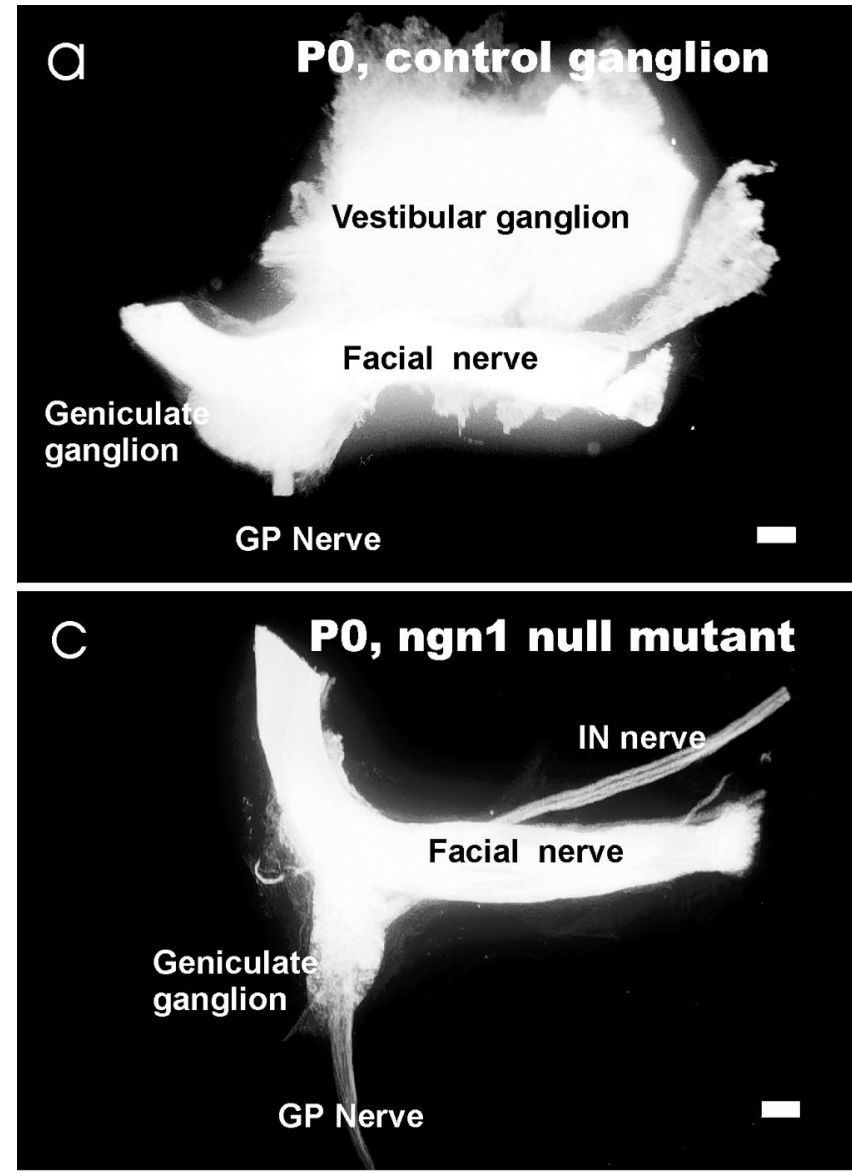

FIG. 6. Dil-labeled ganglia and whole-mounted brains of $P 0(\mathbf{a}, \mathbf{c})$ and E12 embryos (b, d) show the neuronal differences in ngn 1 null mutants (c, d) and control animals (a, b). Dorsal is up, anterior is to the left. Note that the only sensory neurons present in the vestibular and facial nerves of ngn1 null mutants (c) are distal geniculate ganglion sensory neurons with fibers going to the greater petrosal nerve (GP nerve) and chorda tympani (not shown). In contrast, a larger geniculate ganglion and a vestibular ganglion are present in wildtype control littermates (a). The facial nerve is about the same size, as is the intermediate nerve (IN nerve), although it is invisible against the

\section{DISCUSSION}

Ngn1 null mutants suggest autonomy of innerear sensory hair cell development

O ur data confirm and extend findings from a previous report on ngn 1 null mutants ( $M$ a et al. 1998) and show that no sensory neurons project to the ear in E12, E14, E16, and P0 mutants. In addition, we show that the ear of ngn 1 null mutants does not receive any afferent, efferent, or autonomic innervation between E12 and P0. Thus, these mutants permit observation of the differentiation of the ear and its sensory hair cells in vivo in the absence of any innervation.

O ur data also agree with numerous'in vitro studies ( van de Water 1983; Sobkowicz 1992; Rusch et al. 1998;
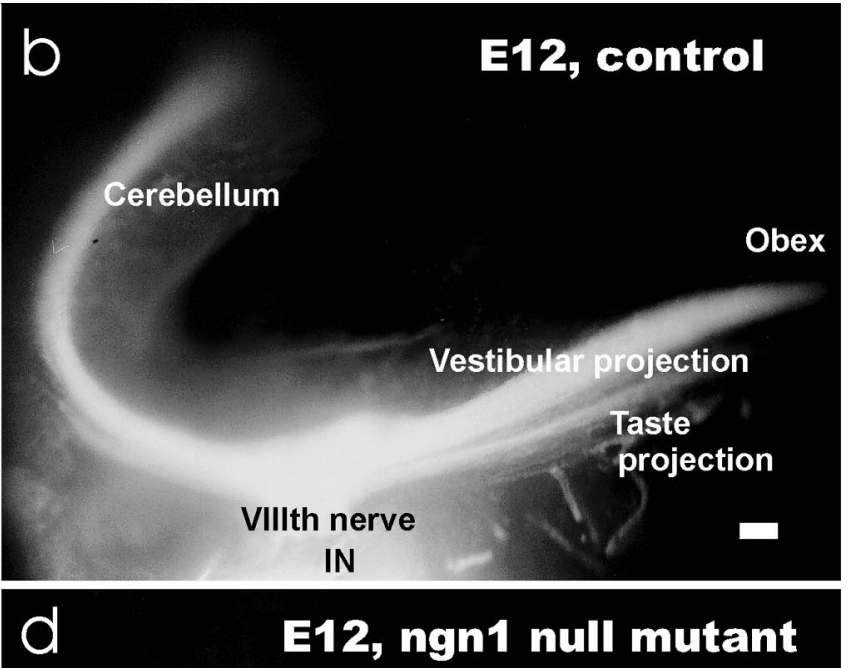

Cerebellum

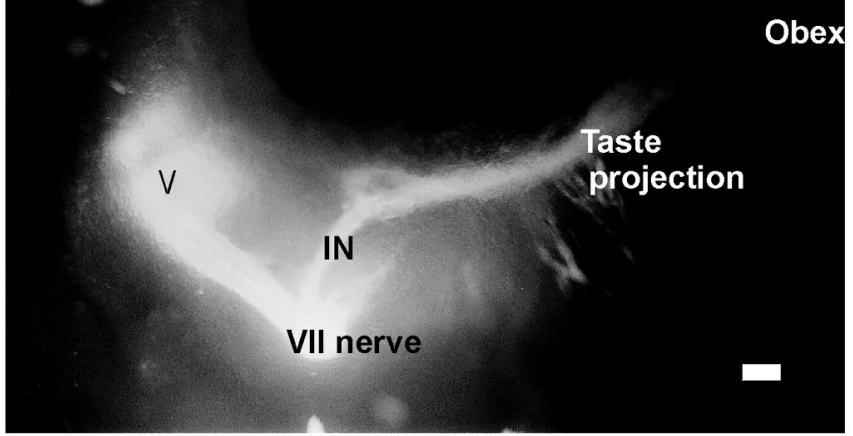

vestibular ganglion in control animals (a). The central projection reflects these differences (right). Thus, both a vestibular projection to the cerebellum and toward the obex and a taste projection just ventral to it are visible in control mice (b). In contrast, only the taste projection to the solitary tract, representing fibers from geniculate sensory neurons and projecting via the intermediate nerve (IN), can be labeled in ngn1 null mutants (d). Facial motoneurons are labeled in both cases but only the ngn 1 null mutants show, in addition, labeled trigeminal motoneurons $(\mathrm{V})$ which project through the facial nerve. Scale bar equals $100 \mu \mathrm{m}$.

Adam et al. 1998), transplantation studies (Swanson et al. 1990), and several studies in neurotrophin and neurotrophin receptor mutants (Ernfors et al. 1995; Fritzsch et al. 1997a, b; Silos-Santiago et al. 1997). The data show that morphological maturation of hair cells is, at least until birth, independent of innervation. In fact, much like some in vitro and neurotrophin mutation studies, we find that even initial formation of presynaptic specializations occurs independent of innervation (Silos-Santiago et al. 1997; Sobkowicz 1992). We also find an initial differentiation of what appears to be two types of vestibular hair cells based on different diameters of stereocilia and kinocilia ( Fig. 5) as recently described (Rusch et al. 1998; Fritzsch et al. 1997a). Further cultivation of these dener vated ears of ngn 1 null mutants in vitro is needed to show that 

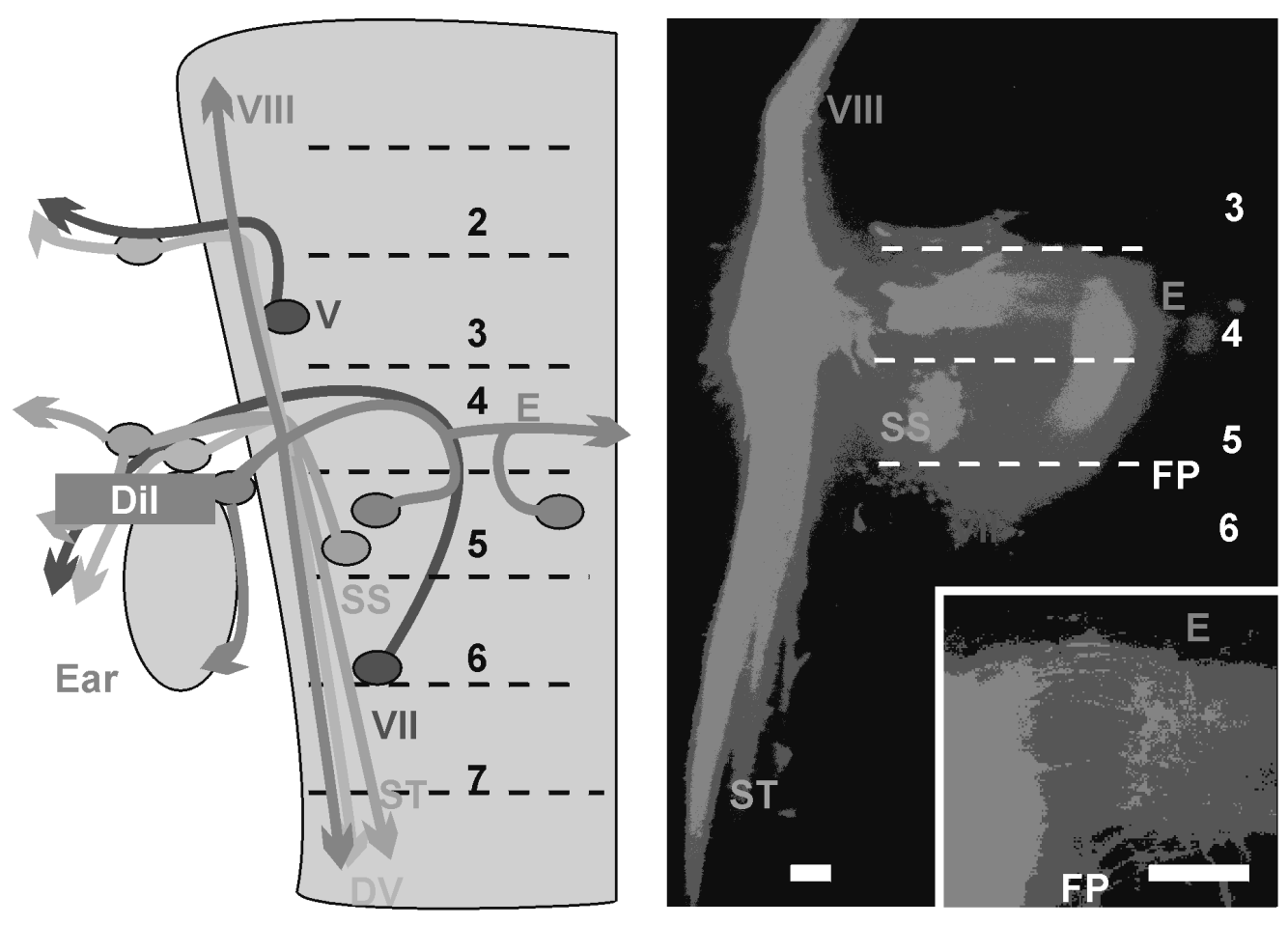

\section{Control E12}
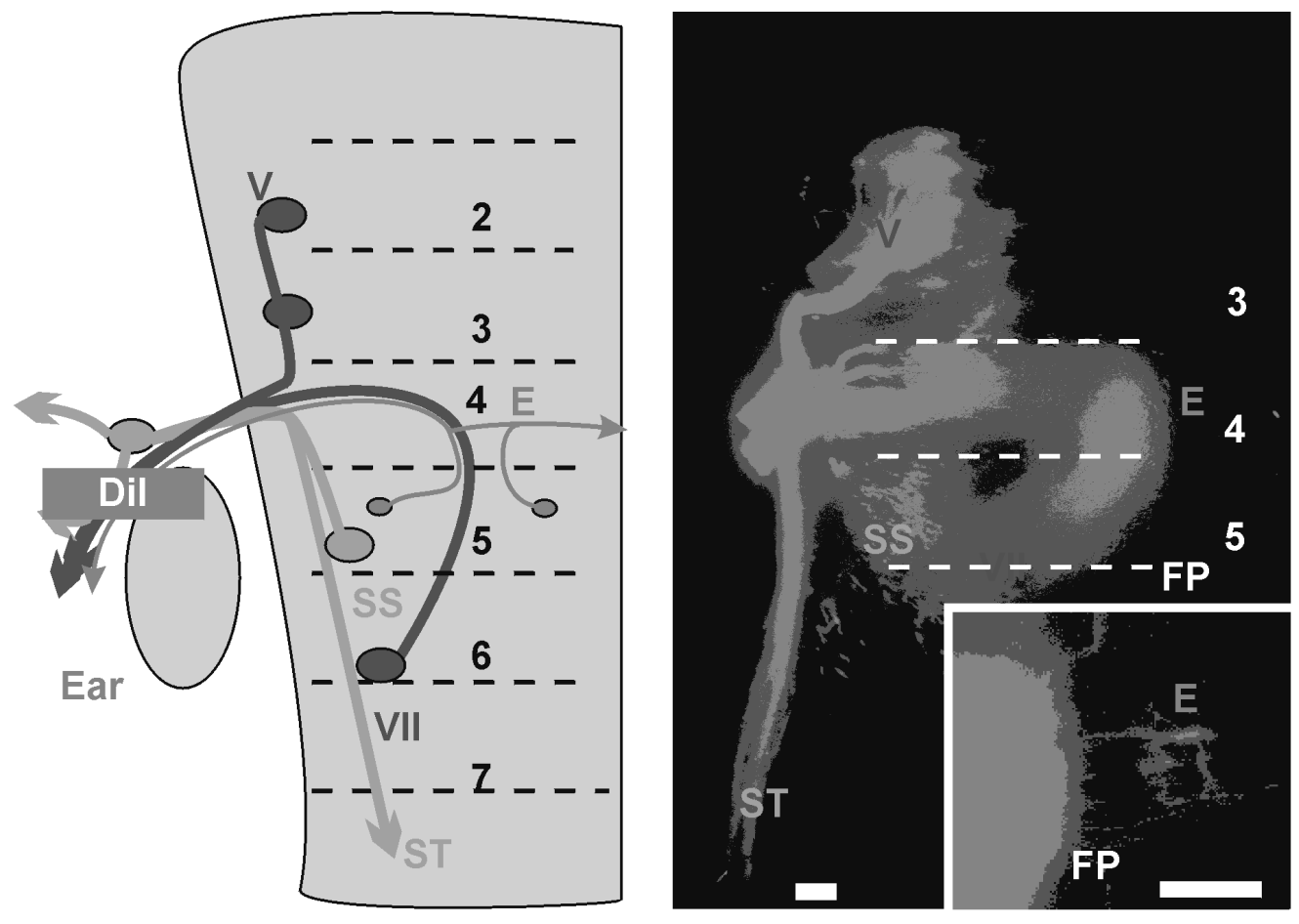

\section{ngn1 null E12}

FIG. 7. Flat mounts (right) show the distribution of motoneurons and sensory projections that can be labeled after application of Dil to the facial and stato-acoustic (VIII) nerves at E12.5. Anterior is to the top. The drawings (left) show the distribution of various cranial nerve afferents and efferents and the placement of the Dil filter strip [not shown in the whole mount (right)]. In control mice, the geniculate ganglion is composed of somatosensory neurons (light blue) and taste sensory neurons (green) which project to the descending tract of $\mathrm{V}$ (DV) and the solitary tract (ST), respectively. Both stato-acoustic (VIII, red) and taste afferents (ST, green) extend along the alar plate in control mice, but only a taste projection to the solitary tract (ST, green) exists in ngn 1 null mutants (bottom). M otor neurons projecting to the facial nerve in control mice are the facial branchial motoneurons (VII, dark blue), extending from rhombomere 4 to rhombomere 6 , and the visceral motoneurons of the superior salivary nucleus (SS; green) in rhombomere 5. Additional neurons are the contralateral and ipsilateral efferents to the ear in rhombomere $4(E$, red; see inserts for detail). Trigeminal motoneurons ( $\mathrm{V}$, dark blue) in rhombomeres 2 and 3 are labeled only in ngn 1 null mutants after Dil application to the facial nerve. $\mathrm{FP}=$ floor plate, arabic numerals indicate rhombomeres, scale bar $=100 \mu \mathrm{m}$. 

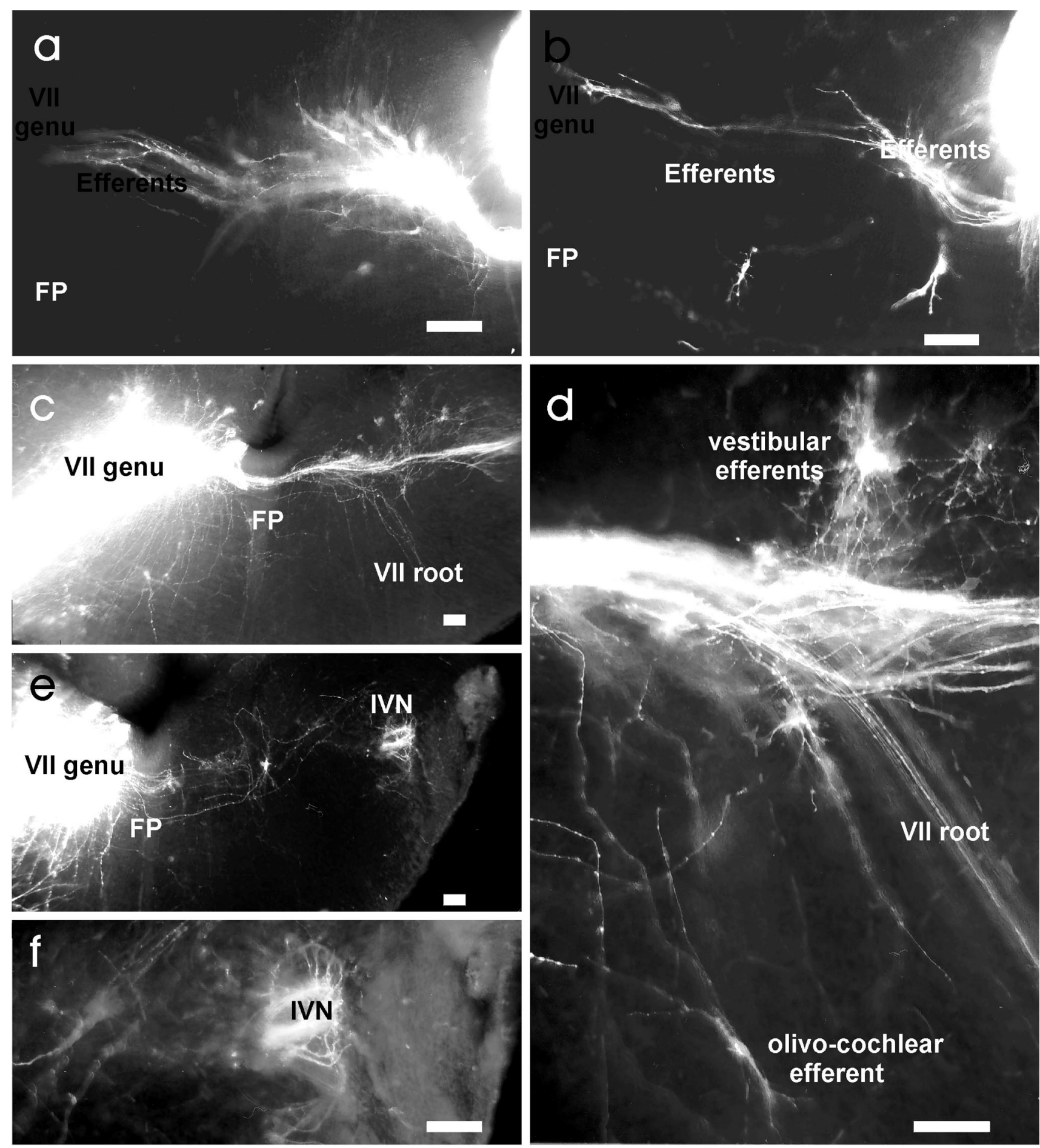

FIG. 8. Images show $80-\mu \mathrm{m}$ - thick sections approximately through rhombomere 4 of E12 wildtype control (a), E12 ngn1 null mutant littermates (b), and E16 ngnl null mutants (c-f). Dil application was to the facial nerve (ngn1 null mutants) or the facial nerve and inner ear combined (control). Note that fewer contralateral efferent cells of a similar distribution as the inner ear efferents in control mice can

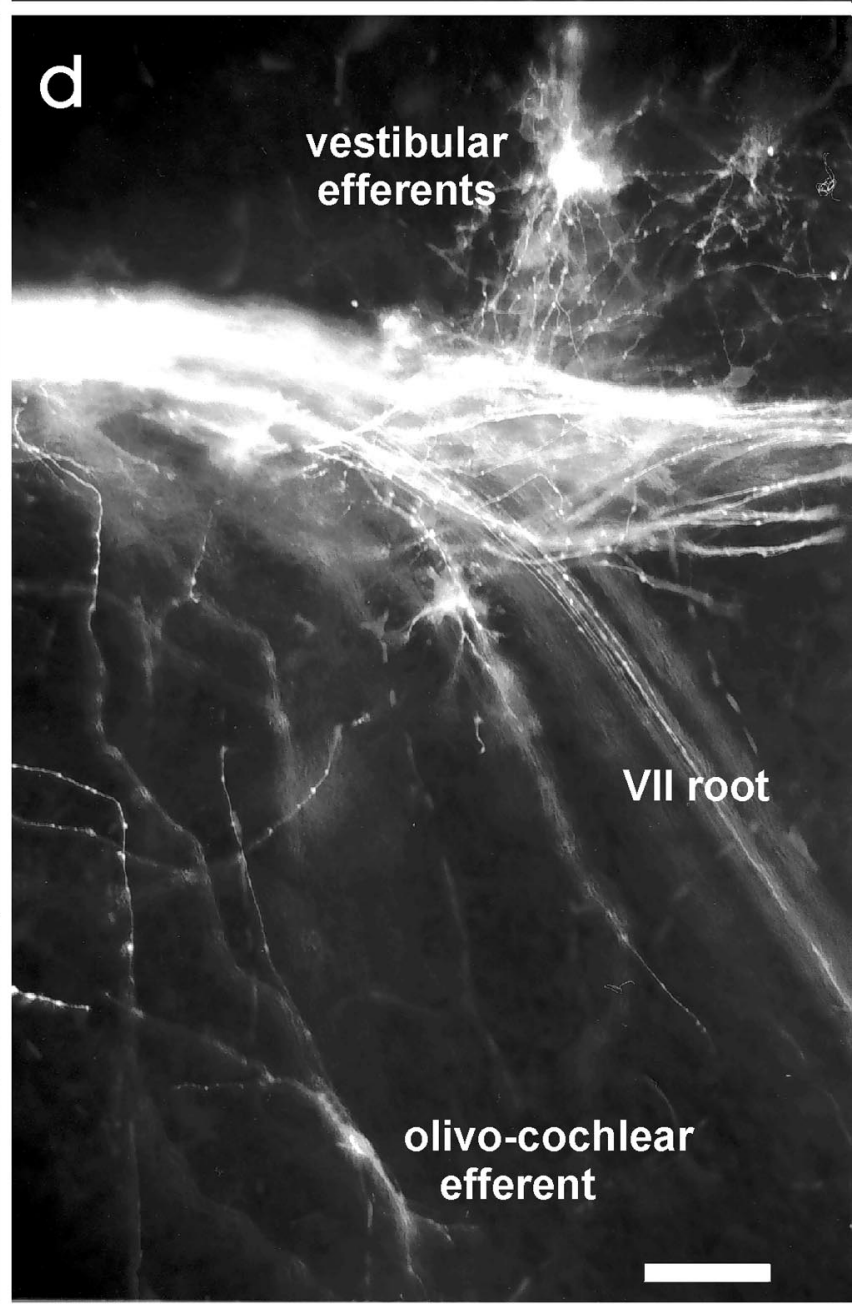

be labeled in the ngn 1 null mutants $(\mathbf{a}, \mathbf{b})$. These contralateral efferents are found at E16 in the position of vestibular efferents and the superior olive $(\mathbf{c}, \mathbf{d})$. Contralateral fibers of these cells can be traced to the inferior vestibular nucleus [IVN, $(\mathbf{e}, \mathbf{f})$ ] where they appear to terminate. Some contralateral fibers appear to exit through the contralateral facial nerve root (d). FP $=$ floor plate, scale bar equals $100 \mu \mathrm{m}$.

continuous functional development, like the morphological maturation we showed in the"present study, is fully independent of innervation (Rusch et al. 1998). Alternatively, these data may show some deviation from

normal physiology in denervated ears as found in tissue culture of chicken ears (Sokolowski et al. 1993).

Reduction in length or width of vestibular sensory epithelia in ngn 1 null mutants also occurs in Math1 
null mutants where no hair cellsform (Bermingham et al. 1999). H owever, no quantitative data were provided concerning the possible shortening of the cochlea, and it is unclear whether the reduction in size is exclusively related to the absence of hair cells in $M$ ath 1 null mutants. Compared with the single atonal gene in fruit flies, two mammalian atonal orthologs are used for inner-ear development: one for hair cells (M ath1) and one for sensory neurons (ngn 1 ). Atonal null mutant flies lose all chordotonal sensory organs (Sun et al. 1998), a loss that is only partly mimicked by ngn 1 null mutants (loss of all sensory neurons, some hair cells in smaller sensory epithelia). The mammalian atonal homolog, M ath1, seems to affect only sensory hair cell formation, but null mutants show a normal development of supporting cells in smaller sensory epithelia (Lysakowski et al. 1999; Bermingham et al. 1999). No information is as yet available on the pattern of innervation in $M$ ath 1 null mutants. Interestingly, the mind bomb zebrafish mutant (Haddon et al. 1998) forms ten times more hair cells in larger sensory epithelia, forms no supporting cells, and shows a twofold increase in sensory neurons. Thus far these zebrafish mutantshave been analyzed only with respect to DeltaNotch signaling, a system that is used to regulate expression of proneuronal genes to specific progenitors, but not with respect to the initial pattern of proneuronal gene expression (Sun et al. 1998). It is possible that mind bomb upregulates $M$ ath 1 and ngn 1 simultaneously in all possible precursors, overriding the lateral inhibition of the Delta-N otch signaling system, comparable to fly mutants and frog mutants that overexpress proneuronal genes (Sun et al. 1998; Ma et al. 1996). Mouse mutants that constitutively overexpress ngn 1, Math1, or both are needed to test these suggestions.

Neither any possible ngn $1 /$ Math 1 interaction nor the clonal relationship of sensory neuron and sensory hair cell precursors are fully resolved in mice. Analysis of transcription factorsknown to regulate proneuronal genes (Chitnis 1999; Lyden et al. 1999) could help to fill that gap. Data in mice suggest that at least some sensory neurons become postmitotic and differentiate while inside the sensory epithelium primordium (Bruce et al. 1997). Data on chicken development cannot rule out the possibility that some clonal relationship of sensory hair cells and sensoryneurons may exist only very early in ear development (Fekete et al. 1998). Sensory neurons start to delaminate at stage 13 ( 48 hours of incubation; Adam et al. 1998), whereas the earliest labeling of clones may not have happened before stage 20 ( 72 hours of incubation; Fekete et al. 1998). We suggest that the ear is forming clones that become progressively more restricted later in development.

It appears that progenitor cells that depend on ngn 1 die in ngn 1 null mutants ( $M a$ et al. 1999b; Anderson 1999). Consequently, progeny of these cells will not form. If these lost progenitors are not only forming sensory neurons but also are giving rise to some hair cell and supporting cell clones, smaller sensory epithelia with fewer hair cells will form. For example, in the saccule, progenitors with the largest capacity to form both sensory neurons and various cells of the inner ear initially depend on ngn 1 . If these progenitors die, the entire saccule would be reduced in size; precisely what we found. More data are needed on the detailed expression of Prospero, Numb, and Delta-Notch homologs in mammals to evaluate this scenario.

Ngn1 null mutants redirect trigeminal motoneurons and inner-ear efferent projections

The projection of sensory neurons into the brainstem of vertebrates is organized into longitudinal columns:

1. the visceral sensory projection to the solitary tract,

2. the somatic sensory projection to the descending trigeminal tract, and

3. the inner-ear projection to the vestibular and cochlear nuclei of the brainstem (Fritzsch and Northcutt 1993; Nieuwenhuys et al. 1997).

This organization of the central projection allows unequivocal identification of sensory afferent specificity, even if the sensory neurons are distributed in mixed ganglia or some are missing in mutants. Our data show that only visceral sensory projections to the solitary tract via the intermediate nerve remain in the facial and stato-acoustic nerves of ngn 1 null mutants (Fig. 5). Moreover, our data correlate with previous work on trkB null mutants (Fritzsch et al. 1997c). That data suggested that some sensory neurons of the geniculate ganglion are derived from neural crest and only their central projections to the descending trigeminal tract survive in trkB null mutants. In contrast, the more distal, epibranchial, placode-derived sensory neurons of the geniculate ganglion undergo a rapid degeneration in trkB null mutants and the projection to the solitary tract is lost (Fritzsch et al. 1997c). These neural-crest-derived sensory neurons, together with their central projection to the descending trigeminal tract, are missing in ngn 1 null mutants, but the more distal, epibranchial, placode-derived sensory neurons are present and project to the solitar y tract (Figs. 6 and 7).

O ur data show that motoneurons in rhombomeres 2-5 apparently form normally in ngn 1 null mutants, likely because of functional redundancy of $n g n 1$ and ngn 2 in the basal plate ( $M a$ et al. 1997). However, in the absence of sensory neuron formation in the trigeminal and stato-acoustic ganglia, their respective 
cranial nerves do not form. As a result, these motoneurons extend their axons through the still-present facial nerve. This observation is consistent with previous experimental work in chickens (Moody and Heaton 1983) and some mutant mice (Lee et al. 1994) which showed that trigeminal motoneuronscannot reach the periphery in the absence of trigeminal sensory neurons as a substrate to grow along. O ur data and those of others (Fritzsch and Northcutt 1993) suggest that the trigeminal motoneuron projection through the facial nerve may be related to the inability of trigeminal motor axons to extend to the periphery without a sensory component. However, some minor misrouting of trigeminal motoneurons into the facial nerve has also been reported in $\mathrm{H}$ oxbl misexpressing chickens (Bell et al. 1999). In these cases, the misrouting has been related to the misexpression in the hindbrain rather than to the unattached trigeminal ganglion observed in some cases. Since no data are available on changed expression of homeobox genes in ngn 1 null mutations, we cannot evaluate this suggestion further. Absence of trigeminal neuron labeling in E16 and neonatal ngn 1 null mutants suggests that this trigeminal projection is transitory. It is possible that trigeminal motoneurons will degenerate in the absence of a proper target that can sustain them (Oppenheim 1991).

Previous work has shown that inner-ear efferents derive from facial branchial motoneurons during development (Fritzsch 1999) and, like the facial branchial motoneurons, should form normally in ngn 1 null mutant mice. In the absence of inner-ear afferents, efferent neurons are apparently unable to reach the ear and some seem to project into the facial nerve, as indicated by the labeling of contralateral cells in rhombomere 4 (Figs. 7 and 8). These suggestions are in agreement with data on otocyst extirpations in chicken which showed that inner-ear efferent cells develop normally, migrate to the contralateral side, and project through the facial nerve (Fritzsch 1999). Others have shown that facial motoneurons can be misrouted after otocyst manipulation (von Bartheld et al. 1992). Thus, efferents and facial motoneurons, if challenged by the absence of an inner ear in chicken and ngn 1 null mutant mice, have some capacity to reroute (Figs. 7 and 8). In chicken and other vertebrates, inner-ear efferents and facial motoneuron axons diverge outside the brain (Hellmann and Fritzsch 1996; Fritzsch et al. 1993). In the brainstem in mice, however, the inner-ear efferents and facial motoneuron axons are separate al ready (Bruce et al. 1997; Fritzsch 1999). It is possible that because of this anatomical peculiarity, fewer inner-ear efferent neurons are able to reach the facial nerve in ngn 1 null mutant mice than in otocyst-deprived chicken. The tracing data of the facial nerve in E16 ngn 1 null embryos show what appear to be contralateral efferent cells that are in their topographically correct positions (Bruce et al. 1997; Fig. 8). We also traced fibers of these cells to the vestibular nuclei, where they ended in the inferior vestibular nucleus (Fig. 8) as recently described (Benson and Brown 1996). It is possible that some efferent cells will remain in neonates but project only to the inferior vestibular nuclei and no longer to the facial nerve. Further tracings in ngn 1 null mutants are necessary to validate this suggestion.

\section{ACKN O W LED G MENTS}

This work was supported by NIDCD (2P01 DC00215-14A1; $B F)$, the $H$ oward Hughes Medical Institute (DA), and an NIMH Center Grant (PI, H . Lester, DA, Q M) . Dr. D.H . Nichols helped with the English; Mrs. M. Christensen and Ms. C. Miller provided excellent technical assistance. We express our gratitude to two referees whose detailed criticism helped to improve the manuscript, and to Dr. D. Fekete for her comments on parts of this paper.

\section{REFEREN CES}

Adam J, Myat A, LeRoux I, Eddison M, Henrique D, ISH-H orowicz D, LEWIS J. Cell fate choices and the expression of Notch, Delta and Serrate homologues in the chick inner ear: parallel with Drosophila sense-organ development. Development 125:46454654, 1998.

ANDERSON DJ. Lineages and transcription factorsin the specification of vertebrate primary sensory neurons. Curr. O pin. Neurobiol. 9:517-524, 1999. DOI: 10.1016/ S0959-4388 (99) 00015-X.

ALTMAN J, BAYER S. Development of the cranial nerve ganglia and related nuclei in the rat. Adv. Anat. Embryol. Cell Biol. 74:190, 1982.

Bell E, Wingate RJT, Lumsden A. Homeotic transformation of rhombomere identity after localized Hoxb1 misexpression. Science 284:2168-2171, 1999.

Benson TE, Brown MC. Synapses from medial olivocochlear branches in the inferior vestibular nucleus. J. Comp. Neurol. 372:176-188, 1996. DOI: 10.1002/ ( SICI) 1096-9861 (19960819) $372: 2<176::$ AID-CNE2 > 3.0.CO;2-0.

Bermingham NA, Hassan BA, Price SD, Vollrath Ma, Ben-Arie N, EATock RA, Bellen HJ, LySAKowsKı A, Zoghbı HY. Math1, an essential gene for the generation of inner ear hair cells. Science 284:1837-1840, 1999.

Bruce LL, KingsLey J, Nich ols DH, FritzSCH B. The development of vestibulo-cochlear efferents and cochlear afferents in mice. Int. J. Dev. Neurosci. 15:671-692, 1997. DOI: 10.1016/ S0736-5748 (96) 00120-7.

CARNEY PR, SILVER J. Studies on cell migration and axon guidance in the developing distal auditory system of the mouse. J. Comp. Neurol. 215:359-369, 1983.

CHITNIS AB Control of neurogenesis-lessons from frogs, fish and flies. Curr. O pin. Neurobiol. 9:18-25, 1999. DOI: 10.1016/ S09594388 (99) 80003-8

Cormin JT, Cotanche DA. Development of location-specific hair cell stereocilia in denervated embryonic ears. J. Comp. Neurol. 288:529-537, 1989. 
ERnfors P, VAN DE WATER T, LoRing J, JaEnisch R. Complementary roles of BDNF and NT-3 in vestibular and auditory development. Neuron 14:1153-1164, 1995.

FeKeTE DM, MuthukUmar S, Karago Geos D. H air cellsand supporting cells share a common progenitor in the avian inner ear. J. Neurosci. 18:7811-7821, 1998.

Fode C, Gradwo hl G, Morin X, Dierich A, LeMeur M, Goridis C, GUILLEM OT F. The bH LH protein neurogenin 2 is a determination factor for epibranchial placode-derived sensoryneurons. neurons. Neuron. 20:483-494, 1988.

FRITZSCH B, NICHOLS DH. Dil reveals a prenatal arrival of efferents at developing ears of mice. Hear. Res. 65:51-60, 1993.

FRITZSCH B, NoRTh CUTt RG. Cranial and spinal nerve organization in amphioxus and lampreys. Acta Anat. 148:96-110, 1993.

Fritzsch B, Christensen MA, Nichols DH. Fiber pathways and positional changes in efferent perikarya of 2.5 to 7 day chick embryos as revealed with Dil and dextran amines. J. Neurobiol. 24:1481-1499, 1993.

Fritzsch B, Silos-Santiago I, Bianchi L, Farinas I. Neurotrophins, neurotrophin receptors and the maintenance of the afferent inner ear innervation. Sem. Cell Dev. Biol. 8:277-284 1997a DOI: 10.1006/ scdb.1997.0144.

FRITZSCH B, FARINAS I, REICHARDT LF. Lack of NT-3 causes losses of both classes of spiral ganglion neurons in the cochlea in a region specific fashion. J. Neurosci. 17:6213-6225, 1997b.

Fritzsch B, Sarai PA, Barbacid M, Silos-Santiago I. Mice lacking the neurotrophin receptor trkB lose their specific afferent innervation but do develop taste buds. Int. J. Dev. Neurosci. 15:563-576. 1997c. DOI: 10.1016/ S0736-5748 96 00111-6.

FRITZSCH B, BARBACID M, SILOS-SANTIAGO I. The combined effects of trkB and trkC mutations on the innervation of the inner ear. Int. J. Dev. Neurosci. 16:493-505, 1998a.

FRITZSCH B, BARBACID M, SILOS-SANTIAGO I. Nerve dependency of developing and mature sensory receptor cells. Ann. NY Acad. Sci. 855:14-27, 1998b.

FRITZSCH B, PIRVOLA U, YLIKOSKI J. Making and breaking the innervation of the ear: Neurotrophic support during ear development and its clinical implications. Cell Tissue Res. 295:369-382, 1999. DOI: 10.1007/ s004410051244.

FRITZSCH B. Ontogenetic and evolutionary evidence for the motoneuron nature of vestibular and cochlear efferents. $\mathrm{CI}$ BERLIN The Efferent Auditory System Singular Publishing San Diego 3159, 1999.

HADDON C, JIANG Y-J, SMITHERS L, LEWIS J. Delta-Notch signaling and the patterning of sensory cell differentiation in the zebrafish ear: evidence from the mind bomb mutant. Development 125:46374644, 1998.

Hellmann B, Fritzsch B. Neuroanatomical and histochemical evidence for the presence of common lateral line and inner ear efferents and of efferents to the basilar papilla in a frog, Xenopus laevis. Brain Behav. Evol. 47:185-194, 1996.

LEE JE. Basic helix-loop-helix genes in neural development. Curr. Opin. Neurobiol. 7:13-20, 1997.

Lee K-F, Simon H, Chen H, Bates B, Hung M-C, H auser C. Requirement for neuregulin receptor erbB2 in neural and cardiac development. Nature. 378:394-398, 1994.

Lyden D, Young AZ, Zagzag D, Yan W, Gerald W, O'Ellly R, Bader BL, Hynes RO, Zhuang Y, Manova K, BenezRa R. Idl and Id3 are required for neurogenesis, angiogenesis and vascularization of tumour xenografts. Nature. 401:670-676, 1999.

Lysakowski A, Bermingham NA, Price SA, Vollrath MA, Hassan B, Ben arie N, Hellen HJ, Eatock RA, Zoghbi HY. Math1 null mutants fail to develop hair cells but do develop supporting cells. ARO Abstr. 22:176, 1999.

Ma Q, KinTNer C, ANDERSON DJ. Identification of neurogenin, a vertebrate neuronal determination gene. Cell. 87:43-52, 1996.
Ma Q, Sommer L, Cserjesi M, Anderson DJ. Mash1 and neurogenin 1 expression patterns define complementary domains of neuroepithelium in the developing CNS and are correlated with regions expressing notch ligands. J. Neurosci. 17:3544-3652, 1997.

Ma Q, Chen Z, Del Barco-Barrantes I, de la Pompa Jl, Anderson DJ. Neurogenin 1 is essential for the determination of neuronal precursors for proximal cranial sensory ganglia. Neuron. 20:469482, 1998.

MA Q, ANDERSON DJ, FRITZSCH B. ngn 1 mutants have no afferent, efferent and autonomic inner vation of the ear but develop normal hair cells in smaller sensory epithelia. ARO Abstr. 22:176, 1999a.

Ma Q, Fode C, Guillemot F, Anderson DJ. Neurogenin 1 and neurogenin2 control two distinct waves of neurogenesis in developing dorsal root ganglia. Genes Dev. 13:1717-1728, 1999b.

MOODY SA, HEATON MB. Developmental relationships between trigeminal ganglia and trigeminal motoneurons in chick embryos. III. Ganglion perikarya direct motor axon growth in the periphery. J. Comp. Neurol. 213:350-354, 1983.

Nieumenhuys R, Ten donkelaar HJ, Nicholson C. The central ner vous system of vertebrates. Springer-Verlag Berlin 2200, 1997

OpPenheIm RW. Cell death during development of the nervous system. Ann. Rev. Neurosci. 14:453-503, 1991.

RUBEN RJ. Development of the inner ear of the mouse: a radioautographic study of terminal mitosis. Acta Oto-Laryngol. 220:1-44, 1967.

RusCh A, LySAKowsKı A, EATock RA. Postnatal development of type I and type II hair cells in the mouse utricle: acquisition of voltagegated conductances and differentiated morphology. J. Neurosci. 18:7487-7501, 1998.

Schimmang T, Minichiello L, Vazquez E, San jose I, Giraldez F, KLEIN R, REPRESA J. Developing inner ear sensory neurons require trkB and trkC receptorsfor innervation of their peripheral targets. Development. 121:3381-3391, 1995.

Shibamori Y, Tamamaki N, Saito H, Nojyo Y. The trajectory of the sympathetic fibers to the rat cochlea as revealed by anterograde and retrograde WGA-H RP tracing. Brain Res. 646:223-229, 1994.

Silos-Santiago I, Fagan AM, Garber M, Fritzsch B, Barbacid M. Severe sensory deficits but normal CNS development in newborn mice lacking trkB and trkC tyrosine protein kinase receptors. Eur. J. Neurosci. 9:2045-2056, 1997

So BKowICZ HM. The development of innervation in the organ of Corti. RomAnd R Development of Auditoryand Vestibular Systems 2. Elsevier Amsterdam 59-100, 1992.

So kO LOWSKI BH A, STAHL LM, FUCHS PA. Morphological and physiological development of vestibular hair cells in the organ-cultured otocyst of the chick. Dev. Biol. 155:134-146, 1993 DOI: 10.1006/ dbio.1993.1013.

SUN Y, JAN LY, JAN YN. Transcriptional regulation of atonal during development of the Drosophila peripheral ner vous system. Development 125:3731-3740, 1998.

SWANSON GJ, HowARD M, LEWIS J. Epithelial autonomy in the development of the inner ear of a bird embryo. Dev. Biol. 137:243257, 1990.

Studer M, Lumsden A, Ariza-McNaughton L, Bradley A, KrumLAUF R. Altered segment identity and abnormal migration of motor neurons in mice lacking Hoxb-1. Nature. 384:630-634, 1996.

VAN DE WATER TR. Embryogenesis of the inner ear: "In vitro studies". Rom AND R Development of Auditory and Vestibular Systems. Academic Press New York 337-374, 1983.

Vetter DE, Liberman MC, Mann J, Barhanin J, Boulter J, Brown MC, Saffiote-Kolman S, Heinemann SF, Belen Elgoymen A. Role of $\alpha 9$ nicotinic receptor subunits in the development and function of cochlear efferent innervation. Neuron. 23:93-103, 1999.

von Bartheld CS, Yang W, Rubel EW. Normal and abnormal 
pathfinding of facial nerve fibers in the chick embryo. J. Neurobiol. 23:1021-1036, 1992.

Walsh E, MCGee J, MCFadden S, Liberman M. Longterm effects of sectioning the olivocochlear bundle in neonatal cats. J. Neurosci. 18:3859-3869, 1998.

Wiechers B, Gestwa G, Mack A, Carroll P, Zenner H-P, Knipper M. A changing pattern of Brain-Derived Neurotrophic Factor expression correlates with rearrangement of fibers during cochlear development of rats and mice. J. Neurosci. 19:30333042, 1999.

Zuo J, Treadaway J, Buckner TW, Fritzsch B. Visualization of $\alpha$ 9 acetylcholine receptor expression in hair cells of transgenic mice containing a modified bacterial artificial chromosome. Proc. Natl. Acad. Sci. 96:14100-14105, 1999. 